\title{
Comparison of Radiation Damage in Silicon Induced by Proton and Neutron Irradiation
}

\author{
A. Ruzin, G. Casse, M. Glaser, A. Zanet, F. Lemeilleur, and S. Watts
}

\begin{abstract}
The subject of radiation damage to silicon detectors induced by $24-\mathrm{GeV} / c$ protons and nuclear reactor neutrons has been studied. Detectors fabricated on single-crystal silicon enriched with various impurities have been tested. Significant differences in electrically active defects have been found between the various types of material. The results of the study suggest for the first time that the widely used nonionizing energy loss (NIEL) factors are insufficient for normalization of the electrically active damage in case of oxygen- and carbon-enriched silicon detectors. It has been found that a deliberate introduction of impurities into the semiconductor can affect the radiation hardness of silicon detectors.
\end{abstract}

Index Terms - Neutrons, protons, radiation damage, radiation hardness, silicon detectors.

\section{INTRODUCTION}

$\mathbf{S}$ ILICON detectors operated in a harsh radiation environment, such as high-energy physics experiments, nuclear reactors, and space, sustain a significant lattice damage. The damage is induced mostly by heavy ions, protons, neutrons, and pions. Photons are considerably less damaging. Some of the radiation-induced damage is electrically active, altering the operation of the detectors. For the device, the most challenging changes are an increase of the leakage current and a variation in the full depletion voltage. The leakage current increases because of the introduction of generation centers and the full depletion voltage changes because of the introduction of space charge. It has been suggested that the formation of $\mathrm{V}_{2} \mathrm{O}$ centers after irradiation contributes to the space charge of the depleted detector volume [1], [2]. The centers act as deep-level acceptors and therefore introduce negative space charge in the depleted silicon. The models predict partial suppression of $\mathrm{V}_{2} \mathrm{O}$ centers in silicon enriched by oxygen because of the enhanced formation of A-centers, $\mathrm{VO}$, resulting in a lower rate of space-charge formation and therefore improved radiation hardness. A low concentration of carbon, which is known to getter silicon self-interstitials, has also been predicted by the model to have beneficial

Manuscript received December 8, 1998; revised March 4, 1999 and June 7, 1999. This study was conducted in the framework of the CERN RD-48 (ROSE) Collaboration.

A. Ruzin was with CERN EP Division, CH-1211 Geneva 23, Switzerland. $\mathrm{He}$ is now with the Department of Physical Electronics, Tel Aviv University, 69978 Tel Aviv, Israel.

M. Glaser, A. Zanet, and F. Lemeilleur are with CERN EP Division, CH1211 Geneva 23, Switzerland.

G. Casse was with CERN EP Division, CH-1211 Geneva 23, Switzerland. He is now with the University of Liverpool, L69 7ZE Liverpool, U.K.

S. Watts is with the Department of Electrical Engineering and Electronics, Brunel University, Uxbridge, UB83PH, U.K.

Publisher Item Identifier S 0018-9499(99)08141-1. effect on the radiation hardness. However, the model failed to quantify adequately the increase in volume current in the case of irradiation by hadrons. A more recent model attributes a significant fraction of current generation and space-charge density, in case of neutron irradiation, to charge exchange between traps inside the clusters [3].

Numerous studies have been carried out on radiation hardness of silicon detectors, using various irradiating particles. The widely used nonionizing energy loss (NIEL) factor was frequently applied to normalize bulk radiation damage effects induced by various particles to that induced by $1-\mathrm{MeV}$ neutrons [4]-[9].

\section{EXPERIMENT}

In this study we compare the electrically active damage induced by $24-\mathrm{GeV} / c$ protons from the PS at CERN to the damage induced by neutrons from a Triga-type nuclear reactor. ${ }^{1}$ The theoretical "relative hardness" of the $24-\mathrm{GeV} / c$ protons, which is the ratio of the NIEL factors of the $24-\mathrm{GeV} / \mathrm{c}$ protons to that of $1-\mathrm{MeV}$ neutrons, can be roughly extrapolated to a value 1-1.2 from [4] or to a value $0.5-0.7$ from [5] and [6]. The neutrons in the nuclear reactor core have a wide energy spectrum ranging from thermal to around $10 \mathrm{MeV}$, with a total relative hardness factor of $\sim 0.88$ [10].

All the detectors reported in this study are p-i-n structures with $5 \mathrm{~mm} \times 5 \mathrm{~mm}$ contacts and single guard rings. The thickness of the various float-zone (FZ) bulk materials is 260 to $310 \mu \mathrm{m}$. The "intrinsic" region was initially n-type with resistivity of $1.3-2.5 \mathrm{k} \Omega \cdot \mathrm{cm}$. All detectors reported on in this work were processed by ITE [11]. Initial volume currents at full depletion voltage of all reported detectors are in the range of $1-3 \mu \mathrm{A} / \mathrm{cm}^{3}$. The oxygen was introduced by a jet of gas during the FZ process [12]. The concentrations of oxygen and carbon were measured by SIMS at EVANS [13]. In the standard silicon material the concentration of oxygen is below the background level of $4 \times 10^{16} \mathrm{~cm}^{-3}$ and the concentration of carbon $\sim 9 \times 10^{15} \mathrm{~cm}^{-3}$, close to the carbon background concentration. The impurity background concentrations are measured with an FZ reference sample, in which the concentrations are very low and known. In the oxygen-enriched silicon the concentration of oxygen is $2.1 \times 10^{17} \mathrm{~cm}^{-3}$ and the concentration of carbon $\sim 10^{16} \mathrm{~cm}^{-3}$. A relatively high concentration of carbon, $1.8 \times 10^{16} \mathrm{~cm}^{-3}$, and a low concentration of oxygen (below the background level) have been obtained in FZ silicon by diffusion from Czochralski $(\mathrm{Cz})$ polycrystalline wafers. First, a small piece of graphite

\footnotetext{
${ }^{1}$ TRIGA reactor at Jozef Stefan Institute, Ljubljana, Slovenia.
} 


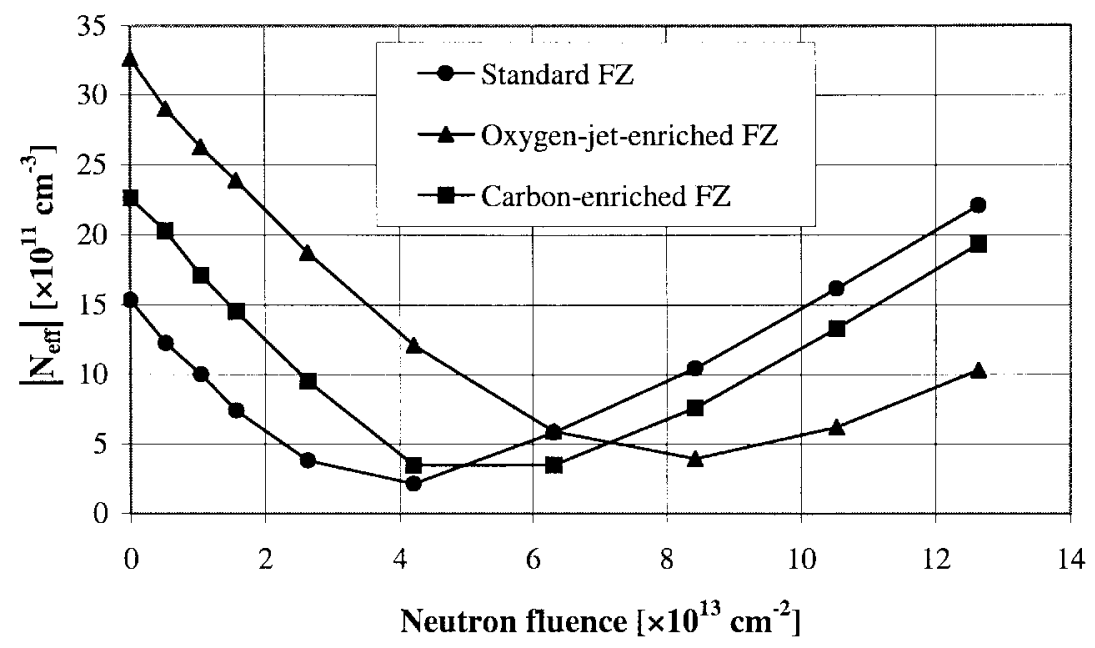

Fig. 1. Dependence of $\left|N_{\text {eff }}\right|$ on neutron fluence.

was melted in silicon polycrystalline. Then, a wafer of this carbon-enriched polysilicon was placed between a standard FZ polysilicon ingot and the seed. Finally, a standard FZ growth process was performed [14].

The detectors were irradiated in steps, each step followed by a short thermal annealing and then $I-V$ and $C-V$ characterization. The full depletion voltage was evaluated by $C-V$ measurement.

The following paragraph gives a brief explanation of the thermal annealing; a more detailed discussion can be found in [15]. Some radiation-induced defects, such as vacancies and self-interstitials, diffuse and form various combinations among themselves as well as with impurities. Such evolution takes place even below room temperature and results in a corresponding evolution of electrical properties of the devices. The fluxes in proton and neutron irradiations could be significantly different, resulting in different irradiation periods. In order to eliminate the influence of self-annealing during the irradiation and during the radioactivity cooling period, we performed short thermal annealing $\left(4 \mathrm{~min}\right.$ at $\left.80{ }^{\circ} \mathrm{C}\right)$ which simulates about ten days at room temperature (RT). It has been found that following such thermal annealing or ten days at RT, the full depletion voltage reaches a "plateau value," at which it stays for the following 20 days at RT, therefore allowing stable and comparable measurements.

\section{RESUlTS AND DisCUSSION}

A typical variation of the effective space-charge density in the depleted detector, $\left|N_{\text {eff }}\right|$, as a function of the neutron fluence (not normalized to 1-MeV neutrons) is shown in Fig. 1. The density has been calculated from the full depletion voltage $V_{\mathrm{FD}}$ according to

$$
\left|N_{\mathrm{eff}}\right|=\frac{2 \cdot \varepsilon_{0} \cdot \varepsilon_{S i} \cdot V_{\mathrm{FD}}}{q \cdot d^{2}}
$$

where $q$ is the electron charge, $d$ is the effective thickness of the detector, $\varepsilon_{0}$ is the vacuum permittivity, and $\varepsilon_{\mathrm{Si}}$ is the relative permittivity constant of silicon. At a certain fluence, called the inversion fluence, the average space charge in the depleted region becomes negative. The inversion fluence, corresponding to the minimum value of $\left|N_{\text {eff }}\right|$, is directly proportional to the space-charge density before irradiation, as was previously observed [16]. The slope $\beta$, which is the rate at which $\left|N_{\text {eff }}\right|$ increases with fluence after the inversion point, is rather similar for all three types of detectors. The slightly lower value of $\beta$ in the case of oxygen-enriched material is probably due to the proximity of the experimental results to the inversion point. The leakage currents were measured at full depletion voltage and have been found to increase linearly with the fluence for all detector types. Before depletion, in particular for low fluences, the currents increase linearly with the square root of bias voltage, indicating generation current from the depletion region. The volume current is fitted with

$$
I_{\mathrm{vol}}=I_{\phi 0}+\alpha \cdot \phi
$$

where $I_{\phi 0}$ is the volume current before irradiation, $\phi$ is the fluence, and $\alpha$ is the coefficient of introduction of generation centers. The coefficient $\alpha$, in the case of irradiation by neutrons, is similar for the three detector types, $\alpha=$ $(3.85 \pm 0.2) \times 10^{-17} \mathrm{~A} / \mathrm{cm}$.

The typical dependence of $\left|N_{\text {eff }}\right|$ on particle fluence in the case of irradiation by $24 \mathrm{GeV} / c$ protons is shown in Fig. 2 . The three detector types are from the same wafers as the detectors described in Fig. 1. In order to make the comparison as accurate as possible, special care was taken regarding the number of steps before inversion fluence, and regarding the fluence step. In this way, the total amount of annealing at each fluence is similar in both irradiations. It is clearly seen that the slope above the inversion fluence is significantly different for each detector type. The strictly linear dependence of the inversion fluence on the initial space-charge density is violated in this case. The lowest $\beta$ factor has been obtained for oxygen-enriched material and the highest for carbon-enriched material. The $\beta$ coefficient of the oxygen-enriched detector is approximately a factor 3 lower compared to the standard FZ silicon detector, and that of carbon-enriched is a factor 2 to 3 higher. The current-voltage behavior is similar to that observed in neutron irradiation, indicating the validity of the generation mechanism in the depletion region. The increase in the leakage current with fluence remains linear and the slope is the same for all detector types, $\alpha=(2.67 \pm 0.2) \times 10^{-17} \mathrm{~A} / \mathrm{cm}$. 


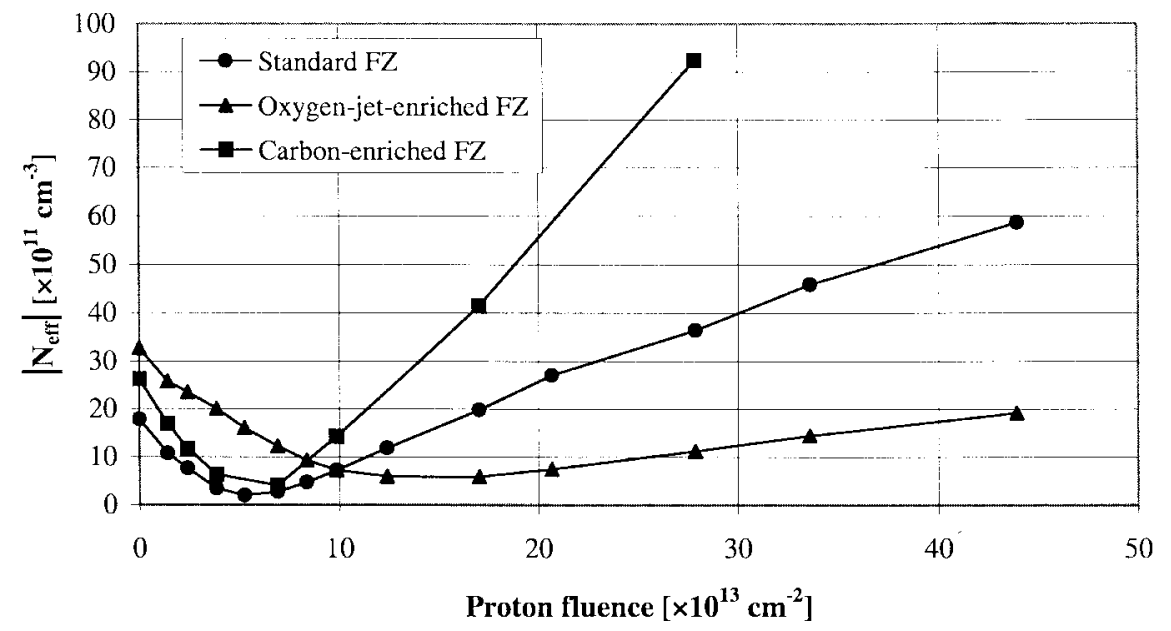

Fig. 2. Dependence of $\left|N_{\text {eff }}\right|$ on proton fluence.

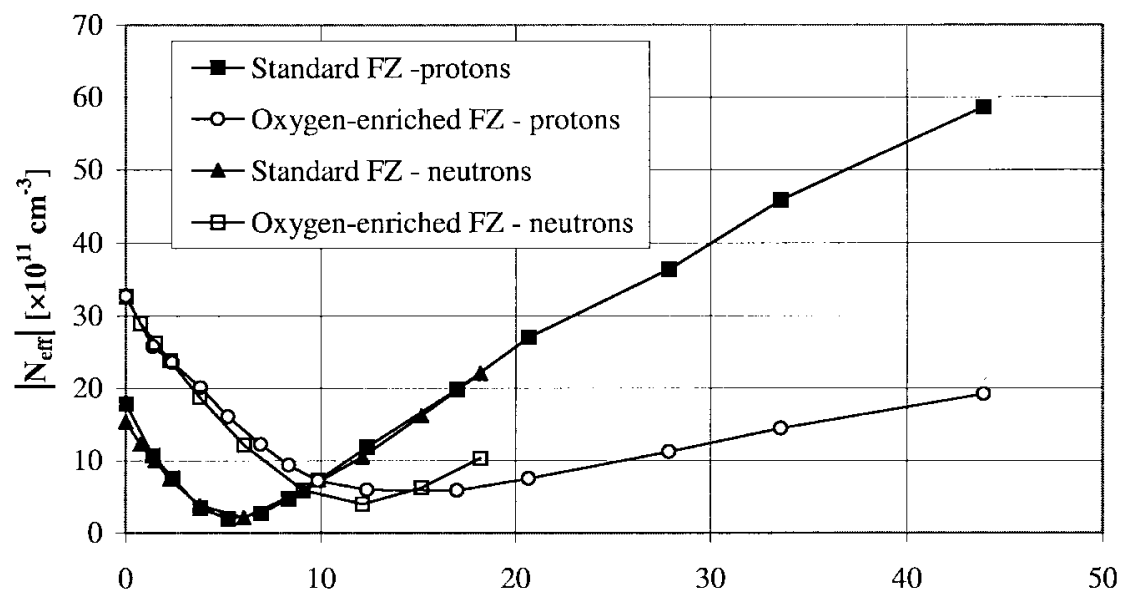

Fluence normalized to $24 \mathrm{GeV} / \mathrm{c}$ protons by $\alpha$ ratio $\left[\times 10^{13} \mathrm{~cm}^{-2}\right]$

Fig. 3. Dependence of $\left|N_{\text {eff }}\right|$ on normalized fluence of protons for standard and oxygen-jet-enriched materials. Normalization of the neutron fluences according to the $\alpha$ factor ratio.

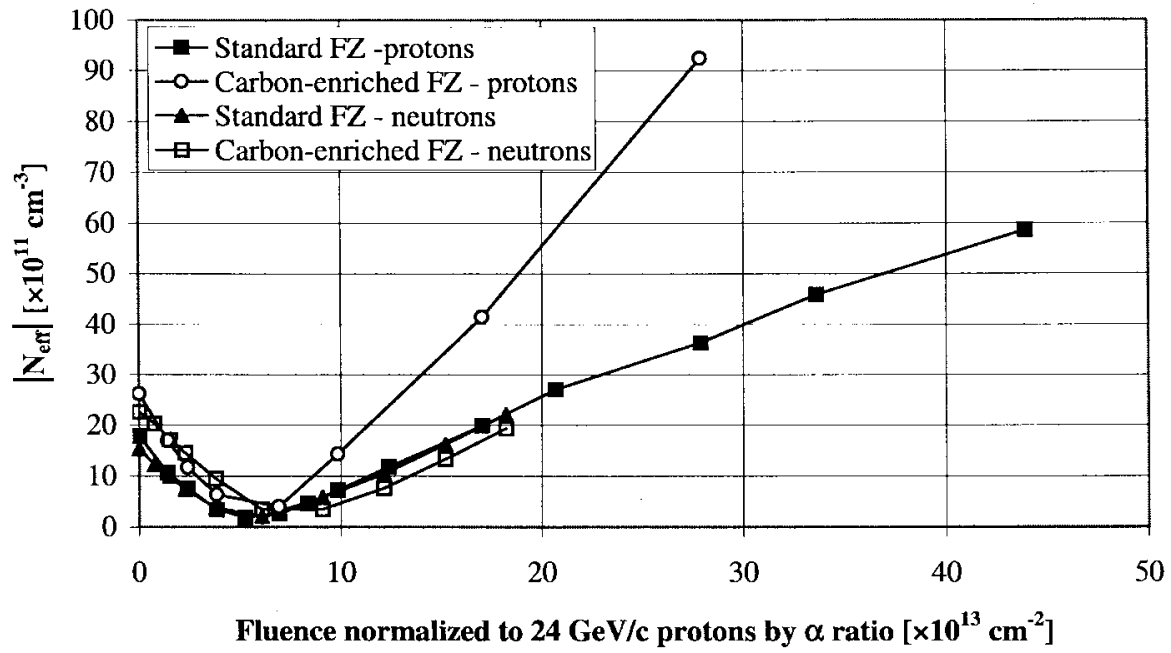

Fig. 4. Dependence of $\left|N_{\text {eff }}\right|$ on normalized fluence of protons for standard and carbon-enriched materials. Normalization of the neutron fluences according to the $\alpha$ factor ratio.

A comparison of the results achieved with protons and neutrons strongly suggests that the electrically active damage cannot be simply normalized by NIEL factors for nonstandard silicon detectors. It can be deduced that the damage introduced by neutrons is not identical in character to that introduced by protons. For instance, the charged particle is bound to have Coulomb interactions with silicon with a much smaller energy transfer, resulting in a larger amount of point-like 
defects. Furthermore, the dependence of the volume current on fluence has been found to be insensitive to the detector type. This implies that the added impurities do not react with the generation centers, yet form complexes that suppress deep acceptor formation or generate compensating positive charge. The cluster model [3] strongly correlates the current and space-charge variations, a correlation which is not observed experimentally in the case of carbon- and oxygen-enriched materials. It is very likely that in the case of hadron-induced damage, the current is generated mostly in the clusters. If, on the other hand, the space-charge formation is also clusterdominated, then in addition to the A-centers, oxygen should form compensating positively charged levels. Further study of defects in oxygen- and carbon-enriched materials is essential in order to fully understand this behavior.

As mentioned above, the $\alpha$ factor represents a rate of introduction of generation centers. If we calculate the ratio of $\alpha$ factors of reactor neutrons to that of $24-\mathrm{GeV} / c$ protons, we get $3.85 / 2.67=1.44$. This ratio is in agreement with the NIEL factor ratio of [4], [5], and [10], as stated above.

The superimposed results of standard, oxygen-enriched, and carbon-enriched detectors on a normalized scale are shown in Figs. 3 and 4. The fluence scale corresponds to $24-\mathrm{GeV} / \mathrm{c}$ protons and the neutron fluences have been normalized by the $\alpha$ factor ratio. The curves of the standard detectors irradiated with protons and neutrons practically coincide. It should be noted that the ratio has been obtained from the current, but the result indicates that similar ratio would have been obtained for the $\beta$ factor and for the inversion point ratio. On such a normalized scale the $\beta$ factor of oxygen-enriched material is a factor $\sim 3$ lower for protons compared to neutrons, in contrast to the $\beta$ factor of carbon-enriched material, which is a factor $\sim 3$ higher for protons compared to neutrons. The effect of the irradiating particle type on inversion point for the carbon- and oxygen-enriched materials is also opposite. Namely, on the normalized scale the oxygen-jet-enriched detectors undergo inversion at lower fluence with neutrons than with protons, in contrast to the carbon-enriched detectors that seem to undergo inversion at lower fluence with protons compared to neutrons. The normalization by the $\alpha$ factor or NIEL coefficients is in reasonable agreement for standard silicon detectors but is violated in the case of carbon- and oxygen-enriched silicon.

\section{REFERENCES}

[1] B. C. MacEvoy, G. Hall, and K. Gill, "Defect evolution in irradiated silicon detector material," Nucl. Instrum. Methods, vol. A, no. 374, pp. 12-26, 1996.

[2] M. Moll, H. Feick, E. Fretwurst, G. Lindstrom, and C. Schutze, "Comparison of defects produced by fast neutrons and ${ }^{60} \mathrm{Co}$-gammas in high resistivity silicon detectors using deep-level transient spectroscopy," Nucl. Instrum. Methods, vol. A, no. 388, pp. 335-339, 1997.

[3] K. Gill, G. Hall, and B. MacEvoy, "Bulk damage effects in irradiated silicon detectors due to clustered divacancies," J. Appl. Phys., vol. 82, no. 1, pp. 126-136, 1997.

[4] A. Van Ginneken, "Nonionizing energy deposition in silicon for radiation damage studies," Fermi Nat. Accelerator Lab., Internal Rep. FN-522, Oct. 1989.

[5] E. A. Burke, "Energy dependence of proton-induced displacement damage in silicon," IEEE Trans. Nucl. Sci., vol. 33, pp. 1276-1281, 1986.

[6] G. P. Summers, E. A. Burke, C. J. Dale, E. A. Wolicki, P. W. Marshal, and M. A. Gehlhausen, "Correlation of particle-induced displacement damage in silicon," IEEE Trans. Nucl. Sci., vol. 34, pp. 1134-1139, 1987.

[7] E. Fretwurst, H. Feick, P. M. Glaser, C. Gossling, E. H. M. Heijne, A. Hess, F. Lemeilleur, G. Lindstrom, K. H. Mahlmann, A. Rolf, T. Schulz, and C. Soave, "Reverse annealing of the effective impurity concentration and long term operational scenario for silicon detectors in future collider experiments," Nucl. Instrum. Methods, vol. A, no. 342, pp. 119-125, 1994.

[8] G. P. Summers, E. A. Burke, P. Shapiro, S. R. Messenger, and R. J. Walters, "Damage correlations in semiconductors exposed to gamma, electron and proton radiations," IEEE Trans. Nucl. Sci., vol. 40, pp. 1372-1379, 1993.

[9] G. P. Summers, E. A. Burke, and M. A. Xapsos, "Displacement damage analogs to ionizing radiation effects," Radiation Meas., vol. 24, no. 1, pp. 1-8, 1995

[10] V. Cindro, G. Kramberger, M. Mikuz, and D. Zontar, "Bias-dependent annealing of radiation damage in neutron-irradiated silicon $\mathrm{p}+-\mathrm{n}-\mathrm{n}+$ diodes," Nucl. Instrum. Methods, vol. A, no. 419, pp. 132-136, 1998.

[11] ITE-Institute of Electron Technology, 02-668 Warsaw, Poland.

[12] E. Nossarzewska-Orlowska, Z. Luczynski, R. Kozlowski, Brzozowski, P. Zabierowski, and B. Majerowski, "Characterization of ITME silicon for detectors," in 3rd ROSE Workshop, DESY-Proc.-1998-02, Hamburg, Germany, Feb. 1998.

[13] EVANS EUROPA, Brunel Univ., Uxbridge, Middlesex UB8 3PH, U.K.

[14] B. Sopko, private communication.

[15] A. Ruzin, G. Casse, M. Glaser, R. Talamonti, A. Zanet, and F. Lemeilleur, "Radiation hardness of silicon detectors manufactured on epitaxial material and FZ bulk enriched with Oxygen, Carbon, Tin and Platinum," in Proc.e 6th Int. Conf. Advanced Technology and Particle Physics, Villa Olmo, Como, Italy, Oct. 1998.

[16] S. J. Bates, B. Dezillie, C. Furetta, M. Glasser, F. Lemeilleur, and E. Leon-Florian, "Proton irradiation of silicon detectors with different resistivities," IEEE Trans. Nucl. Sci., vol. 43, no. 3, pp. 1002-1008, 1996. 